\title{
Maintenance of Cognitive Control during and after Walking in Preadolescent Children
}

\author{
ERIC S. DROLLETTE ${ }^{1}$, TAKAYUKI SHISHIDO ${ }^{2}$, MATTHEW B. PONTIFEX ${ }^{1}$, and CHARLES H. HILLMAN ${ }^{1}$ \\ ${ }^{1}$ Department of Kinesiology and Community Health, University of Illinois at Urbana-Champaign, Urbana, IL; \\ and ${ }^{2}$ Sendai National College of Technology, Miyagi, JAPAN
}

\begin{abstract}
DROLLETTE, E. S., T. SHISHIDO, M. B. PONTIFEX, and C. H. HILLMAN. Maintenance of Cognitive Control during and after Walking in Preadolescent Children. Med. Sci. Sports Exerc., Vol. 44, No. 10, pp. 2017-2024, 2012. Objective: The present study evaluated the effects of an acute bout of moderate-intensity treadmill walking on aspects of cognitive control underlying successful academic achievement. Methods: The study used a within-subjects counterbalanced design with a sample of 36 preadolescent children. Cognitive performance was assessed using a modified flanker task and a modified spatial $n$-back task to assess inhibition and working memory, respectively. Results: No changes in task performance were observed while individuals were actively walking or at seated rest across both tasks. However, during the flanker task, increased response accuracy was observed after exercise relative to post-seated rest. Further observation revealed decrements to response accuracy after seated rest relative to baseline. No such effect was observed for the $n$-back task. Conclusions: These findings suggest selective exercise-induced changes to cognitive control for aspects of inhibitory control and attention but not for working memory. Furthermore, the findings suggest that short bouts of exercise may be efficacious for maintaining cognitive performance, which may have implications for scholastic achievement. Key Words: WORKING MEMORY, INHIBITORY CONTROL, COGNITION, ATTENTION
\end{abstract}

$\mathrm{S}$ edentary behavior continues to rise among children. According to the Centers for Disease Control and Prevention, more than $85 \%$ of children who lived within 1 mile of school walked or biked to school regularly in 1969 (13). Today, that figure has dropped to an estimated $31 \%$ (37). Furthermore, because the "No Child Left Behind" act was enacted over a decade ago, time previously allocated toward physical education and recess has been indirectly reduced by $32 \%$ in more than 6000 elementary school districts across the United States (7). Such trends in sedentary behavior contribute to chronic health problems across the lifespan, including decrements in cognition and brain health (20).

Decreasing opportunities for physical activity during school represents a counterproductive measure (33) when considering the growing body of research indicating a positive relation between regular physical activity and scholastic achievement (9), cognition (35), and memory (8). Furthermore, a smaller body of research has demonstrated that even single bouts of physical activity have transient benefits for cognition after the

Address for correspondence: Charles H. Hillman, Ph.D., Department of Kinesiology and Community Health, 317 Louise Freer Hall, 906 South Goodwin Avenue, University of Illinois at Urbana-Champaign, Urbana, IL 61801; E-mail: chhillma@illinois.edu.

Submitted for publication November 2011

Accepted for publication April 2012.

0195-9131/12/4410-2017/0

MEDICINE \& SCIENCE IN SPORTS \& EXERCISE ${ }_{\circledast}$ Copyright (C) 2012 by the American College of Sports Medicine

DOI: 10.1249/MSS.0b013e318258bcd5 cessation of exercise $(21,24)$. In particular, aspects of cognition necessitating greater levels of cognitive control have demonstrated selectively larger benefits after acute exercise (22).

Cognitive control refers to a subset of cognitive operations responsible for goal-directed actions related to the selection, scheduling, maintenance, and coordination of computational processes underlying perception, memory, and action (31). Within the core concept of cognitive control lie three distinct, yet functionally interrelated, processes known as inhibition, working memory, and cognitive flexibility (14). The current study focused on two of these constructs (i.e., inhibition and working memory). Inhibition represents the ability to attend to task-relevant information in the environment while simultaneously gating out distracting or irrelevant elements that may influence decision making and responding (10), whereas working memory describes the ability to represent, maintain, manipulate, and update information on-line for a short period (26).

Of these processes, most research in the acute exercise literature has investigated inhibition in college-age young adults with enhancements of performance being observed after at least $20 \mathrm{~min}$ of exercise $(23,25)$. In particular, both Hillman et al. (23) and Kamijo et al. (25) separately investigated the effect of acute exercise on inhibitory control in response to a modified Eriksen flanker task (17). The Eriksen flanker task modulates inhibitory control by having participants attend and respond to a focal stimulus amid an array of other distracting stimuli. Replicating the findings of Hillman et al. (23), Kamijo et al. (25) observed that after 20 min of moderate-intensity aerobic exercise, event-related brain potential differences were evident with increased amplitude for 
the P3 component, suggesting increased allocation of attentional resources as a result of the single bout of exercise. These findings $(23,25)$ indicate that in young adults, single bouts of moderate-intensity aerobic exercise may be beneficial to cognitive functioning - and, more specifically, cognitive control processes - and further provide a neuroelectric basis for the observed differences in task performance.

Despite these positive relations with inhibitory control, research investigating post-acute exercise effects on other aspects of cognitive control such as working memory has demonstrated a less robust relation with only a few studies demonstrating positive results (6). Recently, a meta-analysis compared the relation of moderately intense acute exercise on behavioral measures (i.e., reaction time and response accuracy) of working memory both during exercise and after exercise (29). Results of 24 select studies revealed a significant relation between acute exercise and task performance. That is, acute exercise was related to poorer accuracy and shorter reaction time across a variety of working memory tasks. As such, the current study aimed to test the effects of acute exercise on working memory using a spatial $n$-back task. The $n$-back task has been previously used to modulate the updating of working memory. Spatial versions of the $n$ back task require participants to remember the location of targets (i.e., one or two spaces back) and respond accordingly.

Given the observed effects of a single bout of exercise on inhibition and working memory in young adults, the investigation into the effect of acute exercise on these aspects of cognitive control in school-age children is of particular importance, because it has implications for policy relating to the removal of physical activity opportunities from the school day. That is, previous research has implicated these aspects of cognitive control as a necessary faculty involved in academic achievement (36). Specifically, academic achievement in the areas of reading, mathematics, and science has been found to depend upon the inhibition of unrelated information and updating of working memory (36). Such findings suggest that acute exercise induced enhancements in inhibition and working memory may relate to improved learning in the academic environment. Although substantially less research has examined the effect of a single bout of exercise on cognition in children, findings from these investigations are consonant with this assertion. That is, after participation in a single bout of exercise lasting at least $20 \mathrm{~min}$, improvements in cognition have been observed for aspects of concentration (5), inhibition and reading (22), and mathematics (19).

Based in part on the relation between single bouts of exercise and enhancements in cognition, and in an effort to improve the health status of children and decrease sedentary behavior, the American Alliance for Health, Physical Education, Recreation and Dance (30) recommends having teachers integrate short exercise bouts throughout the school day, including during classroom learning. However, this recommendation to exercise during classroom learning may inadvertently have a negative effect on cognitive control processes that subserve academic achievement. That is, although a general enhancement in cognitive function has been observed after a bout of exercise, some evidence exists to indicate decreases in cognitive performance during exercise.

Specifically, a meta-regression by Lambourne and Tomporowski (28) analyzed select research in college-age adults by separating findings according to the time of cognitive assessment, exercise mode, and exercise intensity. The analyses revealed that cognitive decrements were largest within the first 20 min of walking or running and that overall cognitive performance improved after the cessation of the bout of exercise (28). Despite these findings, support for the observed deficits during exercise remains inconclusive because of the exclusion of several studies demonstrating an opposing pattern of results (11). Furthermore, Dietrich and Audiffren (15) suggest that the divergent findings during exercise may be due to the level of mental engagement required by the cognitive task, with exercise facilitating low levels of cognitive engagement and impeding high levels of engagement (i.e., cognitive control). To date, the acute in-task exercise literature remains equivocal on this perspective. Research has demonstrated both decrements $(15,32,12)$ and improvements $(11,24,34)$ during exercise for tasks that necessitate cognitive control. Furthermore, the extent to which these changes in cognitive control occurring during exercise generalize to school-age children is as of yet limited (34). Given the relevance of these relations to scholastic performance, further investigation is needed to better understand the effects of a single bout of exercise on the direction and time course of changes in cognition in school-age children.

Thus, this study examined changes in task performance during two cognitive control tasks that were designed to assess inhibition and working memory during and after moderately intense treadmill walking. On the basis of previous literature, decreased cognitive control was expected during walking because of the competing demands for finite cognitive resources. However, after the cessation of the single bout of walking, improvements in task performance on the cognitive control tasks were predicted. Such findings have important implications of cognitive and scholastic achievement, as well as the educational environment and the context of learning.

\section{METHOD}

\section{Participants}

Thirty-six preadolescent children age 9-11 yr (mean age $=$ $9.9 \pm 0.7 \mathrm{yr}, 20$ female) were recruited from the east central Illinois area. The Institutional Review Board of the University of Illinois approved this study. In accordance with the institutional review board, a legal guardian provided written informed consent, and the child participant provided written informed assent. A legal guardian provided health history and demographics information via a questionnaire, which also included questions regarding socioeconomic status (SES). 
Calculation of SES followed a trichotomous index that included the following measures: a) the highest attained level of education for the mother and father, b) participation in free or reduced price lunch at school, and c) number of parents who work full time (4). Furthermore, parents completed the physical activity readiness questionnaire that identified medical conditions, which may be exacerbated by physical activity (39), the ADHD Rating Scale IV, and the modified Tanner staging scales to characterize pubertal timing (38). Lastly, participants were administered a brief IQ test (K-BIT [27]) by trained experimenters.

Prescreening criteria identified eligible participants as those who reported being free of any neurological disorders, had (corrected to) normal vision, scored below the 90th percentile on the ADHD Rating Scale IV, at or below a score of 2 (i.e., prepubescent) on the Tanner staging scales, and demonstrated average or above average intelligence according to the K-BIT. Participants received monetary compensation for participation in the study. Table 1 provides participants' demographics and fitness data.

\section{Cardiorespiratory Fitness Assessment}

Maximal oxygen consumption $\left(\dot{\mathrm{V}}_{2 \max }\right)$ was collected by a computerized indirect calorimetry system (ParvoMedics True Max 2400) with averages of RER and oxygen uptake $\left(\dot{\mathrm{VO}}_{2}\right)$ assessed every $20 \mathrm{~s}$. The test followed a modified Balke protocol (1) on a motor-driven treadmill (Life Fitness 93T Classic; Brunswick Corporation, Schiller Park, IL) at constant speed, with each participant walking for the first 3 min followed by a sustained moderate run. Every $2 \mathrm{~min}$, the incline was sequentially increased $2.5 \%$, and running was sustained until volitional exhaustion was achieved. A Polar HR monitor (model A1; Polar Electro, Finland) was fitted to each participant and used to track HR for the duration of the test. Furthermore, every 2 min, perceived physical effort was measured with a pictographic RPE

\begin{tabular}{|c|c|c|c|}
\hline Measure & All Participants & Female & Male \\
\hline Age (yr) & $9.86(0.7)$ & $9.72(0.7)$ & $10.04(0.67)$ \\
\hline SES & $2.15(0.67)$ & $2.32(0.67)$ & $1.93(0.62)$ \\
\hline Tanner scale & $1.25(0.28)$ & $1.23(0.3)$ & $1.28(0.26)$ \\
\hline BMI $\left(\mathrm{kg} \cdot \mathrm{m}^{-2}\right)$ & $17.4(3.09)$ & $16.8(2.56)$ & $18.16(3.6)$ \\
\hline BMI percentile rank (\%) & $50.8(31.7)$ & $45.4(32.7)$ & $57.7(30.1)$ \\
\hline K-BIT (IQ) & $118.31(12.5)$ & $117.2(9.08)$ & $119.7(15.9)$ \\
\hline WRAT reading & $122.44(15.3)$ & $120(13.15)$ & $125.56(17.65)$ \\
\hline WRAT spelling & 115.47 (16.58) & $112(16.03)$ & $119.88(16.7)$ \\
\hline WRAT arithmetic & $114.47(18.24)$ & $113.7(20.3)$ & $115.5(15.9)$ \\
\hline$\dot{\mathrm{V}} \mathrm{O}_{2 \max }\left(\mathrm{mL} \cdot \mathrm{kg}^{-1} \cdot \mathrm{min}^{-1}\right)$ & $46.28(6.78)$ & $44.52(6.5)$ & $48.48(6.68)$ \\
\hline $\mathrm{V}_{2}$ percentile rank $(\%)$ & $52.36(31)$ & $56.25(33.1)$ & $47.5(28.4)$ \\
\hline Baseline mean HR (bpm) & $89.6(9.8)$ & $89.98(7.9)$ & $89.2(12)$ \\
\hline Walking mean HR (bpm) & $120.4(5.6)$ & $121.5(6.0)$ & $119.1(4.9)$ \\
\hline Seated rest mean HR (bpm) & $89.4(9.3)$ & $88.6(9.3)$ & $90.2(9.6)$ \\
\hline Walking mean OMNI (RPE) & $1.8(0.24)$ & $1.9(0.3)$ & $1.4(0.45)$ \\
\hline
\end{tabular}

SES is defined as "low" if the score is below 2, "moderate" if the score is between 2 and 3 , and "high" if the score is greater than 3. BMI, body mass index; K-BIT, Kaufman brief intelligence test; WRAT, wide range achievement test; baseline mean HR, HR before cardiorespiratory fitness $\left(\mathrm{V}_{2}\right.$ max $)$ test; walking mean $\mathrm{HR}$, average $\mathrm{HR}$ during the acute walking period; seated rest mean HR, average HR during the rest period only; walking mean OMNI (RPE), average RPE during the acute walking period. using the children's OMNI scale (40) that ranged from 0 , "not tired at all," to 10, "very, very tired." The results provided measures of maximal $\mathrm{HR}$ and $\dot{\mathrm{VO}}_{2 \max }$ measured in milliliters per kilogram per minute $\left(\mathrm{mL} \cdot \mathrm{kg}^{-1} \cdot \mathrm{min}^{-1}\right)$. Criterion for achieving $\dot{\mathrm{VO}}_{2 \max }$ was evidenced by achieving two or more of the following: a) a plateau in oxygen consumption evidenced by an increase of less than $2 \mathrm{~mL} \cdot \mathrm{kg}^{-1} \cdot \mathrm{min}^{-1}$ with an increase in workload, b) a peak HR $\geq 185 \mathrm{bpm}$ (1) and an HR plateau (18), c) RER $\geq 1.0$ (2), and/or d) RPE on the children's OMNI scale $\geq 8$ (40).

\section{Cognitive Tasks}

Behavior recordings and stimulus presentation were controlled with Neuroscan Stim software (v 2.0; Compumedics USA, El Paso, TX) on a microcomputer, with each trial presented on a monitor at a focal distance of $1 \mathrm{~m}$. Order for each task was counterbalanced across all participants.

Flanker. A modified Eriksen flanker task (17) was used to assess inhibitory control. Participants were instructed to attend to the center (i.e., target) arrow, while ignoring flanking arrows, and to respond as quickly and accurately as possible with a left button press if the target arrow pointed left and a right button press if the target arrow pointed right. The task included 100 randomized trials with each trial containing an array of five arrows presented for a duration of $200 \mathrm{~ms}$ with a fixed interstimulus interval of $1500 \mathrm{~ms}$. Stimuli consisted of 3 -cm-tall white arrows on a black background. All trials were presented with equiprobable directionality and congruency within congruent (e.g., $<<<<<$ or $>>>>>$ ) and incongruent (e.g., $<<><<$ or $>><>>$ ) arrays (32).

Spatial $\boldsymbol{n}$-back. A modified version of the spatial $n$ back task included six white-framed boxes, each measuring $4 \times 4 \mathrm{~cm}$, spaced in a circular fashion $9.5 \mathrm{~cm}$ from a centrally presented fixation cross. The nature of the $n$-back task is to provide a continuous load on working memory, requiring updating and reorganization of memory representations. For each trial, an illustrated black and white cow appeared pseudorandomly inside one of the six boxes. For the 0-back condition, participants were instructed to respond as quickly and accurately as possible with a right button press when the cow appeared in the upper right box (i.e., target) and with a left button press when the cow appeared in any of the remaining five boxes (i.e., correct reject). For the 1-back and 2-back conditions, participants were instructed to respond as quickly and accurately as possible with a right button press if the cow appeared in the same box as the previous trial (i.e., target) for the 1-back condition and two trials previous for the 2-back condition. A left button press indicated if the cow was in any of the four remaining locations (i.e., correct reject). All trials were presented for a duration of $250 \mathrm{~ms}$ with a fixed interstimulus interval of $2500 \mathrm{~ms}$ on a green (r: $51, \mathrm{~g}: 78, \mathrm{~b}: 20)$ background. Targets were presented with $33.3 \%$ probability, with the 0 -back condition containing 45 trials with 15 targets and the 1- and 2-back containing 72 trials with 24 targets each. 


\section{Procedure}

The protocol used a within-subjects repeated-measures design. Each participant took part in three separate sessions of testing with session two and three designed with repeating cognitive assessments under different conditions. All three sessions occurred during the same time on separate days with an average of 8.4 days (1.6 SE) between tests. Participants were instructed to avoid caffeine and vigorous physical activity before each laboratory visit.

Session 1. Participants began by completing informed consent/assent, followed by the collection of demographic and prescreening information. Next, they received the opportunity to practice each of the cognitive tasks. Practice included 60 trials of the flanker task and 20 trials of each condition of the modified spatial $n$-back task (0-back, 1 -back, and 2-back). All tasks were performed while participants sat in a chair placed atop a motor-driven treadmill located in a private testing chamber. The treadmill was equipped with a sturdy removable response device designed to adjust vertically for height and provide stability during testing. Its ergonomic design mimicked bicycle handlebars with response buttons positioned at thumb placement for both hands. Stimuli were delivered on the computer screen attached to a swing arm and positioned toward the front of the treadmill. After the practice trials, participants completed a $\dot{\mathrm{VO}}_{2 \max }$ test to obtain aerobic fitness measures and maximal HR. Before the $\dot{\mathrm{V}} \mathrm{O}_{2 \max }$ test, participants were fitted with a Polar HR monitor and baseline HR was recorded. Height and weight were also recorded using a stadiometer and a Tanita WB-300 Plus digital scale (Tanita Corporation, Arlinghton Heights, IL). After the $\dot{\mathrm{VO}}_{2 \max }$ test, participants were given a 5-min cool down period, and the session was concluded when HR returned to within $10 \mathrm{bpm}$ of the resting HR.

Sessions 2 and 3. Randomization and counterbalancing of participants occurred before the second session to minimize confounding variables due to learning effects and the order of administration (i.e., seated rest, walking vs. walking, seated rest). Each session had three periods in which cognitive task performance was assessed: before walking or seated rest, during walking or seated rest, and after walking or seated rest. Each period contained a set of cognitive tasks such that upon cessation of both sessions, each participant completed six unique versions of all tasks. Versions of cognitive tasks were counterbalanced across participants with order of task administration remaining standardized within each period beginning with the flanker task, with the $n$-back task immediately after. Short breaks ( $5 \mathrm{~min} 54 \mathrm{~s} \pm 18 \mathrm{~s}$ ) were afforded between periods with an average of $19 \mathrm{~min} 37 \mathrm{~s} \pm 12 \mathrm{~s}$ spent completing each period and $70 \min 48 \mathrm{~s} \pm 30 \mathrm{~s}$ spent completing each session. The postwalking period was not initiated until HR returned to $\pm 10 \mathrm{bpm}$ of baseline. The time between the cessation of the exercise bout and the initiation of the postwalking testing ( $5 \mathrm{~min} 12 \mathrm{~s} \pm 12 \mathrm{~s}$ ) and the cessation of the seated rest and the initiation of the post-seated rest testing $(5 \min 42 \mathrm{~s} \pm 18 \mathrm{~s})$ periods did not differ significantly, $t(34)=$
$1.5, P=0.14$. Participants were tested individually and seated for every period, with the exception of the testing period that occurred while walking.

Before each session, participants were fitted with a Polar HR monitor. HR and RPE using the children's OMNI scale were taken at baseline and after performance on each cognitive task. A second set of practice trials was completed before testing. Sixty percent of maximal HR (determined from the $\dot{\mathrm{VO}}_{2 \max }$ test) was the intensity of the walking condition for each participant. Table 1 provides participants' average HR data during exercise and during seated rest.

\section{Statistical Analysis}

Statistical analyses were conducted on reaction time (RT) and response accuracy for each cognitive task separately using SPSS v.18 (SPSS, Chicago, IL). Analysis for the flanker task followed a 2 (mode: walking, seated rest) $\times 2$ (trial type: congruent, incongruent) $\times 3$ (time: before, during, after) factorial model. Analysis for the $n$-back task followed a 2 (mode: walking, seated rest) $\times 3$ (target: 0-back, 1-back, 2 back) $\times 3$ (time: before, during, after) model. Findings were reported using the Greenhouse-Geisser statistic, with a significance level of $P=0.05$. Further reporting included partial $\eta^{2}$ and estimated effect size for main effects and interactions. Please refer to Table 2 for complete results of each factorial analysis. Significant findings were decomposed using Bonferroni corrected $t$-tests for post hoc comparisons.

TABLE 2. Statistical results for each task and measure.

\begin{tabular}{|c|c|c|c|c|}
\hline Effect & $F$ & $d f 1 / d f 2$ & $P(F)$ & $\eta^{2}$ \\
\hline \multicolumn{5}{|l|}{ Flanker accuracy } \\
\hline Mode & 0.01 & $1 / 33$ & 0.92 & $<0.005$ \\
\hline Congruency & 50.4 & $1 / 33$ & $<0.005$ & 0.60 \\
\hline Time & 0.18 & $1.6 / 52.5$ & 0.79 & $<0.005$ \\
\hline Mode $\times$ congruency & 0.03 & $1 / 33$ & 0.86 & $<0.005$ \\
\hline Mode $\times$ time & 4.7 & $1.4 / 45.9$ & 0.02 & 0.12 \\
\hline Congruency $\times$ time & 3.9 & $2 / 64$ & 0.03 & 0.10 \\
\hline Mode $\times$ congruency $\times$ time & 0.03 & $1.8 / 60.5$ & 0.97 & 0.01 \\
\hline \multicolumn{5}{|l|}{ Flanker reaction time } \\
\hline Mode & 2.9 & $1 / 33$ & 0.10 & 0.08 \\
\hline Congruency & 119.3 & $1 / 33$ & $<0.005$ & 0.78 \\
\hline Time & 2.7 & $1.8 / 59.2$ & 0.08 & 0.08 \\
\hline Mode $\times$ congruency & 0.05 & $1 / 33$ & 0.82 & 0.02 \\
\hline Mode $\times$ time & 2.9 & $1.7 / 58.5$ & 0.07 & 0.08 \\
\hline Congruency $\times$ time & 8.2 & $1.9 / 65.9$ & $<0.005$ & 0.20 \\
\hline Mode $\times$ congruency $\times$ time & 2.96 & $1.9 / 64.3$ & 0.06 & 0.08 \\
\hline \multicolumn{5}{|l|}{$n$-back accuracy } \\
\hline Mode & 2.2 & $1 / 35$ & 0.15 & 0.06 \\
\hline Target & 89.2 & $1.53 / 53.7$ & $<0.005$ & 0.70 \\
\hline Time & 2.8 & $1.9 / 66.5$ & 0.07 & 0.07 \\
\hline Mode $\times$ target & 0.50 & $1.6 / 55.6$ & 0.56 & 0.02 \\
\hline Mode $\times$ time & 0.80 & $1.9 / 67.8$ & 0.43 & 0.02 \\
\hline Target $\times$ time & 2.02 & $3.8 / 132$ & 0.10 & 0.06 \\
\hline Mode $\times$ target $\times$ time & 1.1 & $3.2 / 113.5$ & 0.36 & 0.03 \\
\hline \multicolumn{5}{|l|}{$n$-back reaction time } \\
\hline Mode & 2.4 & $1 / 35$ & 0.13 & 0.06 \\
\hline Target & 111.6 & $1.61 / 56.4$ & $<0.005$ & 0.80 \\
\hline Time & 2.1 & $1.5 / 52.5$ & 0.14 & 0.06 \\
\hline Mode $\times$ target & 0.30 & $1.7 / 61$ & 0.68 & 0.01 \\
\hline Mode $\times$ time & 0.50 & $1.9 / 67.7$ & 0.58 & 0.01 \\
\hline Target $\times$ time & 10.9 & $3.7 / 128.5$ & $<0.005$ & 0.24 \\
\hline Mode $\times$ target $\times$ time & 0.10 & $3.3 / 116.1$ & 0.97 & $<0.005$ \\
\hline
\end{tabular}




\section{RESULTS}

\section{Session Order}

Initial analyses were performed to determine whether session order may have been a confounding variable. Omnibus analyses for the $n$-back task revealed a mode $\times$ time $\times$ session order interaction, $F(2,64)=6.9, P=0.002, \eta^{2}=0.18$. However, decomposition of the interaction did not yield significant findings, $t$ 's $(35) \leq 1.4, P \geq 0.16$. No such effects of session order were observed for the flanker task. Thus, all further analyses were collapsed across session order.

\section{Flanker}

Two participants were eliminated from the flanker analysis. Normality screening of the data set revealed accuracy and reaction time performance that fell three SDs outside the norm in two or more conditions, suggesting that the task instructions were not understood or that the task was too difficult to complete for these individuals. No such normality violations occurred for the $n$-back task; therefore, all participants were included in that analysis.

Response accuracy. The omnibus analysis revealed a main effect of congruency, $F(1,33)=50.4, P<0.005$, $\eta^{2}=0.60$, that was superseded by a congruency $\times$ time interaction, $F(2,64)=3.9, P=0.03, \eta^{2}=0.1$. Post hoc tests indicated greater mean accuracy for congruent trials in the precondition $(95.9 \% \pm 1.0 \%)$ compared with the postcondition $(94.6 \% \pm 1.0 \%), t(33)=2.6, P=0.02$, with further analysis of the congruent trials (before the test $(95.9 \% \pm 1.0 \%)$, during the test $(95.6 \% \pm 0.6 \%)$, and after the test $(94.6 \% \pm$ $1.0 \%)$ ) eliciting greater mean accuracy than incongruent trials (before the test $(91.2 \% \pm 1.5 \%)$, during the test $(90.6 \% \pm$ $1.1 \%)$, and after the test $(91.9 \% \pm 1.1 \%))$ across all three periods, $t$ 's $(33) \geq 4.2, P$ 's $\leq 0.005$. Further analysis revealed a significant interaction of mode $\times$ time, $F(1.4,45.9)=4.7$, $P=0.02, \eta^{2}=0.12$. Decomposition of this interaction revealed a decrease in mean accuracy pre-seated rest $(92 \% \pm 1.3 \%)$ compared with post-seated rest $(94.4 \% \pm$ $1.2 \%), t(33)=2.7, P=0.01$, and compared with postwalking $(94.4 \% \pm 0.8 \%), t(33)=2.7, P=0.01$ (Fig. 1A). Furthermore, participants' response accuracy during the walking session demonstrated no significant changes in the prewalking period, during the walking period, or postwalking periods, $F(1.5,48.6)=1.3, P=0.27, \eta^{2}=0.04$.

Reaction time. The omnibus analysis revealed a main effect of congruency, $F(1,33)=119.3, P<0.005, \eta^{2}=0.78$, that was superseded by a congruency $\times$ time interaction, $F(1.9,65.9)=8.2, P \leq 0.005, \eta^{2}=0.2$. Post hoc tests indicated shorter RT for congruent trials during pretest (532.5 \pm $13.3 \mathrm{~ms})$ compared with posttest $(559.9 \pm 14.1 \mathrm{~ms}), t(33)=$ $3.1, P \leq 0.005$. Further analysis revealed the expected shorter RT for congruent trials across all three periods (before the test $(532.5 \pm 13.3 \mathrm{~ms})$, during the test $(548.9 \pm$ $14.3 \mathrm{~ms})$, and after the test $(559.9 \pm 14.1 \mathrm{~ms}))$ compared with incongruent trials (before the test $(581.3 \pm 13.8 \mathrm{~ms})$, during the test $(585.3 \pm 14.9 \mathrm{~ms})$, and after the test $(589.3 \pm$ $14.6 \mathrm{~ms})), t^{\prime} \mathrm{s}(33) \geq 6.1, P$ 's $\leq 0.005$. No significant effects involving mode were observed, $F$ 's $(1.7,58.5) \leq 2.9, P^{\prime} \mathrm{s} \geq$ $0.07, \eta^{2}=0.08$ (Fig. 1B and Table 2). However, the data also suggest that a speed-accuracy tradeoff may have occurred in the exercise condition across time. Inspection of exercise accuracy measures appears numerically, but not significantly, worse in the precondition relative to the rest condition with accompanying longer RT. Accordingly, planned comparisons were performed to examine whether a correlation was present between RT on correct trials and response accuracy. If a task were associated with a speedaccuracy tradeoff, we should expect to see an increase in accuracy accompanying an increase in RT, thus resulting in a positive correlation. However, no significant effects were observed in all conditions, with the condition during rest having the strongest correlation, $r(34)=-0.28, P=0.1$, with findings occurring in the opposite direction to that of
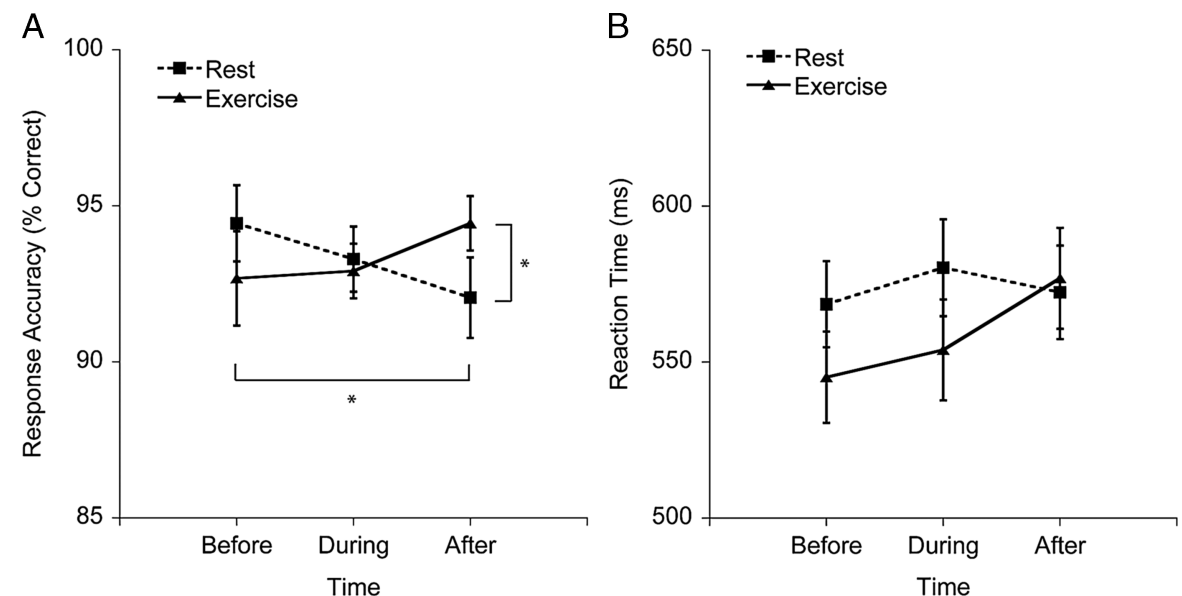

FIGURE 1-Flanker accuracy (A) and reaction time (B). 

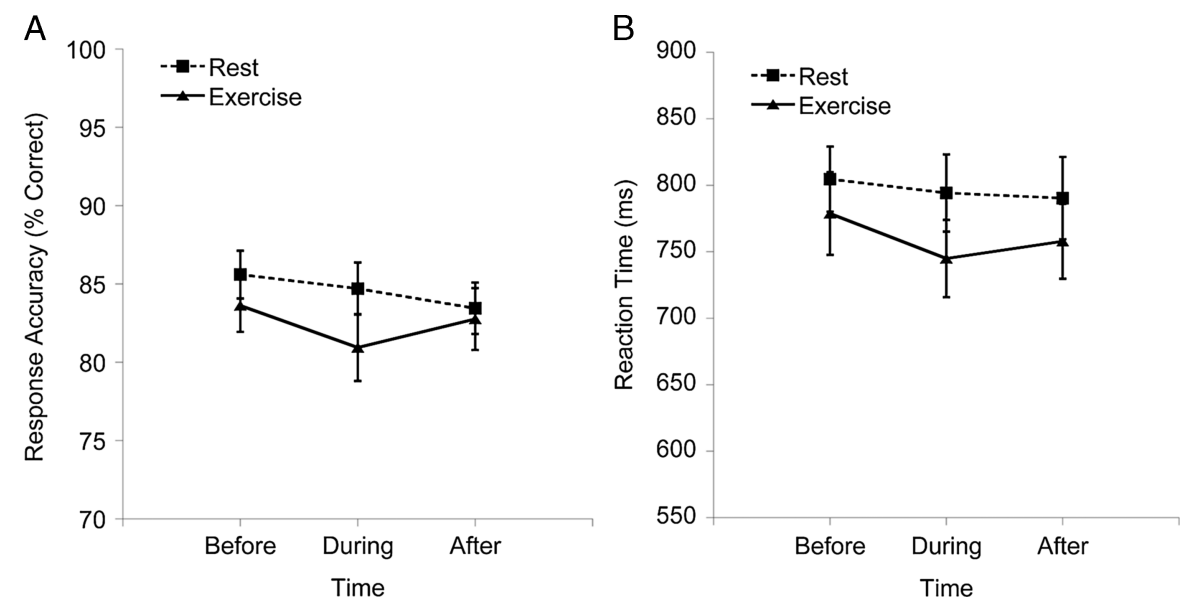

FIGURE 2-n-back accuracy (A) and reaction time (B).

a speed-accuracy tradeoff. A second test was performed to further evaluate the possibility of a speed-accuracy tradeoff by correlating RT on correct trials and commission error rates (i.e., typically, more errors are made by participants who respond more quickly). A negative correlation would indicate the presence of a speed-accuracy tradeoff. Yet again, no significant effects were observed in all conditions, with the condition during the exercise demonstrating the strongest negative correlation, but not significant, $r(34)=-0.23, P=0.18$.

\section{n-back}

Response accuracy. The omnibus analysis revealed a main effect of target, $F(1.53,53.7)=89.2, P \leq 0.005$, $\eta^{2}=0.7$, with mean accuracy for the 0 -back condition $(93.8 \% \pm 0.8 \%)$ greater than the 1 -back condition $(81.8 \% \pm 2 \%)$ and mean accuracy for the 0-back and 1-back conditions greater than the 2-back condition $(70.8 \% \pm 2.6 \%)$, $t^{\prime} \mathrm{s}(35) \geq 7.7, P^{\prime} \mathrm{s} \leq 0.005$. No significant effects involving mode were observed, $F$ 's $(1,35) \leq 2.2, P^{\prime} \mathrm{s} \geq 0.15, \eta^{2}=0.06$ (Fig. 2A and Table 2).

Reaction time. The omnibus analysis revealed a main effect of target, $F(1.611,56.37)=111.6, P \leq 0.005, \eta^{2}=0.8$, that was superseded by a target $\times$ time interaction, $F(3.7,128.5)=10.9, P \leq 0.005, \eta^{2}=0.24$. Post hoc tests indicated increased reaction time for 2-back targets in the pretest period $(944.7 \pm 37.1 \mathrm{~ms}$ ) compared with 2-back targets during the test $(853.2 \pm 36.5 \mathrm{~ms})$ and in the posttest period $(853 \pm 37.3 \mathrm{~ms}), t^{\prime} \mathrm{s}(35) \geq 4.6, P$ 's $\leq 0.005$. Further analysis indicated that $0-, 1-$, and 2-back targets differed significantly from each other within each of the three periods as expected (before the test: 0-back ( $593.8 \pm 23.7 \mathrm{~ms}), 1$-back (780.8 $\pm 28.2 \mathrm{~ms}), 2$-back $(944.7 \pm 37.1 \mathrm{~ms})$; during the test: 0-back $(617.1 \pm 26.5 \mathrm{~ms}), 1$-back $(772.6 \pm 30.4 \mathrm{~ms}), 2$-back $(853.2 \pm 36.5 \mathrm{~ms})$; after the test: 0 -back $(627.6 \pm 24.6 \mathrm{~ms})$, 1-back $(782.1 \pm 29.8 \mathrm{~ms}), 2$-back $(853 \pm 37.3 \mathrm{~ms})), t$ 's $(35) \geq$ $5.6, P$ 's $\leq 0.005$. No significant effects involving mode were observed, $F^{\prime}$ s $(1,35) \leq 2.4, P^{\prime} \mathrm{s} \geq 0.13, \eta^{2}=0.06$ (Fig. 2B and Table 2).

\section{DISCUSSION}

The main findings revealed that moderately intense aerobic walking facilitates maintenance of attentional aspects of cognitive control in children over time and does not facilitate or impede working memory. Such findings suggest that physical activity may not detract from the learning process, and at the same time, it provides students with an increased opportunity to engage in health behaviors. Specifically, task performance on the flanker and $n$-back tasks did not differ during exercise relative to during rest, indicating that although improvements in cognition were not realized during physical activity, they also did not deteriorate. Furthermore, selective decrements to response accuracy were revealed for the flanker task after seated rest relative to baseline (i.e., before seated rest) and in comparison with after the bout of walking, indicating that attention and inhibitory control decay over time in response to repetitive cognitive challenge under conditions requiring the individual to stay seated. No such differences were observed for working memory. Accordingly, these data suggest that physical activity does not interfere with working memory and may facilitate maintenance of attention over time in cognitively demanding settings, which has public health implications for the educational environment and the context of learning.

Of particular interest is the lack of significant modulation in the cognitive outcomes during exercise. Specifically, the current findings did not reveal any significant differences in reaction time or response accuracy across both tasks when comparing walking with seated rest. Unlike adults $(11,32)$, children appear to be able to effectively maintain inhibition and the updating of working memory while walking at a moderate intensity. One obvious possible reason for our lack of replication of the prior adult studies might stem from the intensity or duration of the exercise bout. That is, given the paucity of acute in-task exercise research, little evidence exists examining what effects various intensities or durations of exercise have on higher-order cognition or, for that matter, the extent to which these effects may generalize to school-age 
children. As such, our conclusion remains limited because of the consequence of insufficient data investigating a doseresponse relation. However, the current findings appear to validate the recommendation provided by the American Alliance for Health, Physical Education, Recreation and Dance to include short bouts of exercise during classroom learning without risk of impairment to cognitive control (i.e., inhibition and working memory) processes necessary for scholastic achievement.

The second main finding comprises the modulation of cognitive outcomes after the conclusion of the exercise bout. Specifically, the current investigation observed a general maintenance in inhibitory control, with better performance in both congruent and incongruent trials of the flanker task after a bout of exercise compared with postrest. Such a finding provides partial replication of prior research with children because Hillman et al. (22) found selective improvements for the incongruent trials of the flanker task after exercise. One potential explanation for these differences may relate to the time at which the cognitive assessment occurred after the cessation of the exercise bout. Specifically, the study of Hillman et al. (22) included a neuroelectrical assessment after exercise, which required preparation of a high-density electroencephalograph array immediately after the exercise bout. They reported that approximately $25 \mathrm{~min}$ was required from the cessation of the exercise bout to the initiation of the flanker task. The present study only required a 5- to 6-min delay for participants' HR to return to preexercise baseline levels before the flanker task begins. Accordingly, this suggests that attention and inhibitory control may modulate on the basis of the period that has elapsed from the conclusion of exercise to the time of cognitive testing. It should be further noted that upon evaluation of the accuracy and RT data, participants during the walking session had a tendency to respond more slowly during the session, thus potentially facilitating the higher accuracy measures after the walking bout. Future research will need to examine this question to better determine the robustness of the task performance effects.

One possibility for the observed differences in working memory and attentional inhibition in comparison with previous research may be due to the engagement of cognitive control during each testing condition across sessions. That is, in contrast to the existent acute exercise literature, the present study systematically maintained a similar level of cognitive engagement during each condition without intermittently modifying such engagement (e.g., having the participants reading a newspaper or watching an educational video during the rest condition). Such continual engagement of cognitive control has been hypothesized to act as a mental priming mechanism resulting in enhancements in cognition (3). Similarly, findings from training games, which require prolonged activation of cognitive control, have observed improvements in behavioral performance after training (16). Thus, the current investigation may have provided consistent control of extraneous variables related to cognitive engagement between conditions, which therefore may have con- tributed to the observed differences relative to the existing literature. However, such explanations remain speculative at this time with further research required to gain understanding of the general and selective nature of single bouts of exercise on attention and inhibition. Regardless, the current findings demonstrated selectivity for the effects of short duration exercise on aspects of cognitive control, with general maintenance in inhibitory control being observed after exercise in the absence of any effect on working memory.

Although the present investigation provides insight into the relation between walking and aspects of cognitive control in children, certain limitations should be noted. One such limitation is the confined age group. Future research should examine a broader range of school-age children to determine whether the differences found herein and in prior research are a function of age or some other factor. Furthermore, future research may gain additional insight, beyond behavioral outcomes, into the neural mechanisms contributing to the observed differences by incorporating neuroimaging measures. Only a few prior studies have been published previously (32), and to our knowledge, no study has investigated cognition during exercise using neuroimaging tools with children. Lastly, the current study took place in a controlled laboratory with limited social and environmental influences. The likely possibility remains that in an applied scholastic setting, more environmental distractions may lead to poorer performance during the exercise bout.

In conclusion, the current study provides support for school-based interventions aimed at reducing inactivity among young children during school without interference to aspects of cognitive control, which may underlie aspects of scholastic performance. Public schools, more than any other institution in the nation, are intimately coupled with more than 54 million children during their first two decades of life (37). If certain lifestyle behaviors, such as physical activity, are adopted and maintained regularly at a young age, they are more likely to track into adulthood. Therefore, positive experiences with physical activity in the classroom may assist in the effort to reduce inactivity across the lifespan. The present study supports the implementation of structured bouts of physical activity in the classroom, before and during classroom learning, which in turn may assist in decreasing sedentary behavior without cost to the faculties necessary for children to navigate the demands of the scholastic environment. Thus, early intervention aimed at adopting physical activity behaviors among children in school should be a national priority of industrialized nations.

Eric S. Drollette, Takayuki Shishido, Matthew B. Pontifex, and Charles $\mathrm{H}$. Hillman have no professional relations with companies or manufacturers who will benefit from the results of the present study. Furthermore, the results of the present study do not constitute endorsement by the American College of Sports Medicine. This manuscript and the data presented in it were not funded by any federal agency, and therefore, no disclosure is warranted. 


\section{REFERENCES}

1. American College of Sports Medicine. ACSM's Guidelines for Exercise Testing and Prescription. 7th ed. New York (NY): Lippincott Williams \& Wilkins; 2010. p. 60-104.

2. Bar-Or O. Pediatric Sports Medicine for the Practitioner: From Physiologic Principles to Clinical Applications. New York: SpringerVerlag; 1983. pp. 376.

3. Best JR. Effects of physical activity on children's executive function: contributions of experimental research on aerobic exercise Dev Rev. 2010;30:331-51.

4. Birnbaum AS, Lytle LA, Murray DM, Story M, Perry CL, Boutelle KN Survey development for assessing correlates of young adolescents eating. Am J Health Behav. 2002;26:284-95.

5. Budde H, Voelcker-Rehage C, Pietrabyk-Kendziorra S, Ribeiro P, Tidow G. Acute coordinative exercise improves attentional performance in adolescents. Neurosci Lett. 2008;441:219-23.

6. Budde H, Voelcker-Rehage C, Pietrabyk-Kendziorra S, Machado S, Ribeiro P, Arafat AM. Steroid hormones in the saliva of adolescents after different exercise intensities and their influence on working memory in a school setting. Psychoneuroendocrinology. 2010;35:382-91.

7. Center for Education Policy. Choices, Changes, and Challenges: Curriculum and Instruction in the NCLB Era. Washington (DC): Center of Education Policy; 2007. p. 18.

8. Chaddock L, Erickson KI, Prakash RS, et al. A neuroimaging investigation of the association between aerobic fitness, hippocampal volume, and memory performance in preadolescent children. Brain Res. 2010;1358:172-83.

9. Chomitz VR, Slining MM, McGowan RJ, Mitchell SE, Dawson GF, Hacker KA. Is there a relationship between physical fitness and academic achievement? Positive results from public school children in the northeastern United States. $J$ Sch Health. 2009;79:30-7.

10. Davidson MC, Amso D, Anderson LC, Diamond A. Development of cognitive control and executive functions from 4 to 13 years: evidence from manipulations of memory, inhibition, and task switching. Neuropsychologia. 2006;44:2037-78.

11. Davranche K, Hall B, McMorris T. Effect of acute exercise on cognitive control required during an Eriksen flanker task. $J$ Sport Exerc Psychol. 2009;31:628-39.

12. Davranche K, McMorris T. Specific effects of acute moderate exercise on cognitive control. Brain Cogn. 2009;69:565-70.

13. Dellinger AM, Staunton CE. Barriers to children walking and bicycling to school-United States, 1999. MMWR Morb Mortal Wkly Rep. 2002;51:701-4.

14. Diamond A. The early development of executive functions. In: Bialystok E, Craik FM, editors. Lifespan Cognition: Mechanisms of Change. New York: Oxford University Press; 2006. pp. 70-95.

15. Dietrich A, Audiffren M. The reticular-activating hypofrontality (RAH) model of acute exercise. Neurosci Biobehav Rev. 2011;35: 1305-25.

16. Erickson KI, Colcombe SJ, Wadhwa R, Bherer L, Peterson MS, Scalf PE. Training-induced functional activation changes in dualtask processing: an fMRI study. Cereb Cortex. 2007;17:192-204.

17. Eriksen BA, Eriksen CW. Effects of noise letters upon the identification of a target letter in a nonsearch task. Percept Psychophys. 1974;16:143-9.

18. Freedson PS, Goodman TL. Measurement of oxygen consumption In: Rowland TW, editor. Pediatric Laboratory Exercise Testing: Clinical Guidelines. Champaign (IL): Human Kinetics; 1993. pp. 91-113.

19. Gabbard C, Barton J. Effects of physical activity on mathematical computation among young children. J Psychol. 1979;103:287-8.

20. Hillman $\mathrm{CH}$, Erickson KI, Kramer AF. Be smart, exercise your heart: exercise effects on brain and cognition. Nat Rev Neurosci. 2008;9:58-65.
21. Hillman CH, Kamijo K, Scudder MR. A review of chronic and acute physical activity participation on neuroelectric measures of brain health and cognition during childhood. Prev Med. 2011;52: $21-8$.

22. Hillman CH, Pontifex MB, Raine LB, Castelli DM, Hall EE, Kramer AF. The effect of acute treadmill walking on cognitive control and academic achievement in preadolescent children. Neuroscience. 2009;159(3):1044-54.

23. Hillman CH, Snook EM, Jerome GJ. Acute cardiovascular exercise and executive control function. Int J Psychophysiol. 2003;48: $307-14$.

24. Joyce J, Graydon J, McMorris T, Davranche K. The time course effect of moderate intensity exercise on response execution and response inhibition. Brain Cogn. 2009;71:14-9.

25. Kamijo K, Nishihira Y, Higashiura T, Kuroiwa K. The interactive effect of exercise intensity and task difficulty on human cognitive processing. Int J Psychophysiol. 2007;65:114-21.

26. Kane MJ, Engle R. The role of prefrontal cortex in workingmemory capacity, executive attention, and general fluid intelligence: an individual-differences perspective. Psychon Bull Rev. 2002;9:637-71.

27. Kaufman AS, Kaufman NL. Kaufman Brief Intelligence Test Manual. Circle Pines (MN): AGS; 1990. pp. 88-109.

28. Lambourne K, Tomporowski P. The effect of exercise-induced arousal on cognitive task performance: a meta-regression analysis. Brain Res. 2010;13:12-24.

29. McMorris T, Sproule J, Turner A, Hale BJ. Acute, intermediate intensity exercise, and speed and accuracy in working memory tasks: a meta-analytical comparison of effects. Physiol Behav. 2011;102: $421-8$.

30. National Association for Sport and Physical Education. Comprehensive School Physical Activity Programs [Position Statement]. Reston (VA): Author; 2008. pp. 1-10.

31. Norman DA, Shallice T. Attention to action: willed and automatic control of behavior. In Davidson RJ, Schwartz GE, Shapiro D, editors. Consciousness and Self-Regulation: Vol. 4 Advances in Research and Theory. New York: Plenum Press; 1986. p. 1-18.

32. Pontifex MB, Hillman $\mathrm{CH}$. Neuroelectric and behavioral indices of interference control during acute cycling. Clin Neurophysiol. 2007; 118:570-80.

33. Sallis JF. We do not have to sacrifice children's health to achieve academic goals. J Pediatr. 2010;156:696-7.

34. Schaefer S, Lovden M, Wieckhorst B, Lindenberger U. Cognitive performance is improved while walking: differences in cognitivesensorimotor couplings between children and young adults. Eur $J$ Dev Psychol. 2010;7:371-89.

35. Sibley BA, Etnier JL. The relationship between physical activity and cognition in children: a meta-analysis. Pediatr Exerc Sci. 2003; $15: 243-56$

36. St Clair-Thompson HL, Gathercole SE. Executive functions and achievements in school: shifting, updating, inhibition, and working memory. Q J Exp Psychol (Hove). 2006;59:745-59.

37. Story M, Kaphingst KM, French S. The role of schools in obesity prevention. Future Child. 2006;16:109-42.

38. Taylor AF, Kuo FE, Sullivan WC. Coping with ADD: the surprising connection to green play settings. Environ Behav. 2001;33: 54-77.

39. Thomas S, Reading J, Shephard RJ. Revision of the physical activity readiness questionnaire. Can J Sport Sci. 1992;17: $338-45$.

40. Utter AC, Robertson RJ, Nieman DC, Kang J. Children's OMNI scale of perceived exertion: walking/running evaluation. Med Sci Sports Exerc. 2002;34:139-44. 